\title{
Fostering Design Thinking in Transdisciplinary Engineering Education
}

\author{
Jo-Yu KUO ${ }^{\mathrm{a}, 1}$, Xue Ting SONG ${ }^{\mathrm{a}}$, Chun-Hsien $\mathrm{CHEN}^{\mathrm{a}}$ and Chandrakant D. PATEL ${ }^{\mathrm{b}}$ \\ ${ }^{a}$ HP-NTU Digital Manufacturing Corporate Lab, School of Mechanical \& Aerospace \\ Engineering, Nanyang Technological University, Singapore \\ ${ }^{\mathrm{b}}$ HP Labs, Palo Alto, CA, USA
}

\begin{abstract}
Disruptive technologies such as 3D printing, artificial intelligence (AI), and robotics have changed how people think, learn, and work fundamentally. Engineering education must adapt to this digital transformation. There has been increasing interest in integrating design in the engineering curriculum around the world. While traditional problem solving is a linear and structured approach, design thinking is set by a human-centered innovation process which leads to better products and services. This concept is well aligned with the educational vision of transdisciplinary engineering. However, it is challenging to teach the mindset of design thinking for people with various domain knowledge. In this paper, the differences in how industrial designers and design engineers tackle a design project are explained. We intend to share a few successful examples regarding how design methodology captures customer requirements and explores creative solutions in the product development lifecycle within the current engineering curriculum. Also, the user experience research in response to the trend of cyberphysical integration is discussed. Finally, we conclude with the need for a holistic curriculum design in digital manufacturing as a case study to illustrate the role of design thinking for future transdisciplinary engineering education.
\end{abstract}

Keywords. Design thinking, engineering education, transdisciplinary, design methodology, 3D printing, digital manufacturing

\section{Introduction}

Disruptive technologies such as $3 \mathrm{D}$ printing, artificial intelligence (AI), and robotics have changed the way people think, learn, and work fundamentally. The education curriculum needs to be developed and reformed progressively in order to train and upskill the current workforce to embrace this digital transformation. There are many educational philosophies such as undeclared major and STEM (science, technology, engineering, mathematics) for different levels of school and higher education [1]. One main consensus is the emphasis on cross-discipline competence. It is also known as a T-shaped skill. The vertical bar of ' $\mathrm{T}$ ' is your domain knowledge in a single field. The horizontal bar of ' $\mathrm{T}$ ' is your ability to collaborate across disciplines. Thus, a good curriculum structure should centralize the importance of a broad base of general skills and knowledge that support one's domain knowledge. This concept is well aligned with the vision of transdisciplinary engineering [2].

\footnotetext{
${ }^{1}$ Corresponding Author, Mail: jykuo@ntu.edu.sg
} 
At the same time, the use of design thinking strategies has been gaining a lot of traction in academia and various industries. The term 'design' is generic and abstract, leading to a wide audience. Fashion designers and design engineers are both considered as design professionals while the former works towards beautiful visual artworks, and the latter is more analytical. Inevitably, there seem to be inconsistent definitions of 'design thinking' in different disciplines. Nowadays this buzz term is coming mostly from business innovation seminars while it shares a common ground with the heuristics principles in engineering ideation [3]. To our understanding, in general, design thinking helps to make sense of the complex connections between diverse perspectives. For that reason, design thinking encourages collaborations and cross-disciplinary teamwork. Therefore, it has become one of the required skills that foster engineering innovations. The question is how to integrate the ideology of design thinking into curriculum development so the students can be exposed to this designer-ly strategy.

The remainder of this paper adapted the format of design thinking proposed by Brenner, et al. [4]. The first section elaborates on the difference in curriculum approach between engineering schools and design schools. It states the 'Why' of the paper's agenda and considers design thinking as a mindset within transdisciplinary engineering education. The second section focuses on 'How' incorporating design thinking as a method has benefited engineering and manufacturing innovations by presenting examples within a current engineering curriculum. Lastly, we posit that design thinking can continue to augment engineering education in the cyberphysical age.

\section{WHY: Perspectives from engineering schools and design schools}

Historically, engineers and designers used to be segregated in different schools and trained with distinct abilities. Mechanical Engineering (ME) is an academic discipline with a long history. Over time, new academic disciplines appeared to cater to the trend of real-world problems. During the Bauhaus movement, Industrial Design (ID) emerged as a new discipline in response to the industrialization of consumer products and massproduction techniques. These disciplines intertwined gradually which has been reflected in the title of school programs (e.g., mechanical engineering design stream, industrial design engineering). This phenomenon seems to imply a good indication that industrial designers and mechanical engineers would make a great team to solve complex problems. However, interdisciplinary collaboration only succeeds based on a shared understanding of the domain knowledge. This is challenging not only for the practitioner but also the educator. To bring the gap closer, it is beneficial to glimpse the core curriculum developed by the respective schools.

Here, we use an example to illustrate the main differences between engineering education and design education. The two schools explore the design space differently. Imagine a task is assigned to design a $3 \mathrm{D}$ printer. The $\mathrm{ME}$ school emphasizes achieving functionality and feasible technical solutions. The design space would inform the technical restrictions for heating and extruding or the strength test of the filament. On the other hand, the ID school emphasizes capturing human-centered requirements. The first few questions that come to their mind could be: Is the printer designed for a kid or a professional? Is it ergonomically friendly for the user to replace the filament? In other words, ME students are taught to manage risk, and ID students are trained to manage human uncertainty. The level of uncertainty can extend to personal preferences and 
experiences. Hence, compared to ME students, ID students tend to pay more attention to the product appearance.

The traditional linear and structured way of thinking is excellent and efficient when solving simple problems. A simple usage problem of a 3D printer can be: why is my printer not working? This problem can be resolved step by step in the troubleshooting process. The users pinpoint the known problem, list the viable options, evaluate them then select the best outcome. Nevertheless, this problem-solving approach is not ideal for ill-defined problems such as 'why users choose printer A over printer B.' People from a strong engineering background may be unfamiliar working around problems with less technical constraints, resulting in difficulties thinking out of the box. This is where design thinking comes into play. Design thinking is an iterative process to relook into our problems from as many different perspectives as possible. During this abductive thinking process, the original problems are reframed to yield more creative solutions.

The integration of experts' domain knowledge enhanced by design thinking enables both designers and engineers to solve problems, considering perspectives from both design and engineering disciplines (see Figure 1). For example, an industrial design engineering curriculum can evolve from a design curriculum augmented by essential engineering knowledge. Similarly, a mechanical engineering design curriculum possess the foundations of a robust engineering curriculum, supplemented by modules and projects that require students to implement design thinking. Introducing design thinking into the traditional engineering curriculum can enhance the core domain knowledge of engineers. Hence, improving the core curriculum developed by the respective schools allows both practitioners and educators to reap the benefits of a true transdisciplinary design engineering education.

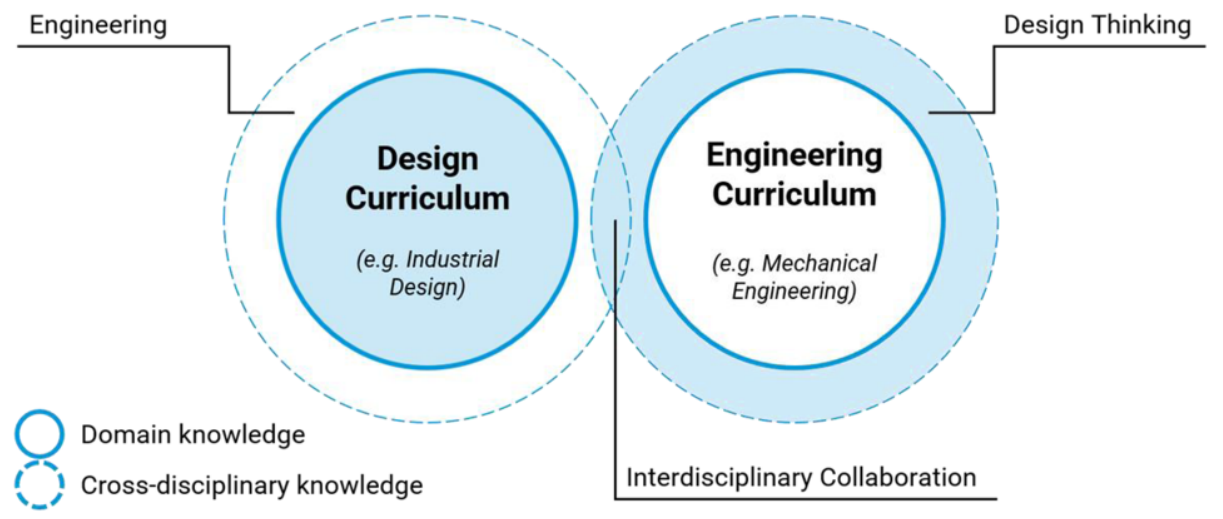

Figure 1. Transdisciplinary design and engineering education.

The rise of 3D printing and digital manufacturing necessitates design thinking within a holistic engineering curriculum. The most wildly used 3D printing process, known as fused deposition modeling (FDM) melts and extrudes the thermoplastic filament through a nozzle layer by layer. This process is fairly slow and often needs to deal with overhangs. In this sense, the next generation of printers may be inspired to minimize the additional support structures so as to reduce the printing time. If one applies linear thinking to solve this problem, he/she might jump to the simple conclusion that we need a more advanced process such as powder bed techniques. Alternatively, following the design thinking approach, the design space can be expanded by having additional degrees of freedom in 
motion to fabricate the 3D model. In a study by Dai, et al. [5], they used a multidirectional deposition method to optimize the toolpath in two steps: first decomposing the volume into sequences of curved surface layers, and then decomposing each surface into the curved toolpath. Their results achieve better precision and surface quality of spatial toolpath generation over the conventional slicer. This appears to be a fine example of design thinking implementation on an engineering problem.

Accordingly, it is not surprising that design thinking has been applied to support the innovations in engineering education [6]. For example, Coleman, et al. [7] developed an instrument based on the similarities between engineers and designers to assess the students' perceived design thinking ability. In an environmental engineering course, Clark, et al. [8] found a positive association between design thinking and the students' perceived creativity and sustainable career practices. Palacin-Silva, et al. [9] reported the lessons learned from the perspectives of teachers and students on running a software engineering capstone project with design thinking. Tomita, et al. [10] proposed a structured framework to guide and trace the exploratory nature of design thinking for complex societal problems in the context of systems engineering. In this paper, we intend to apply design thinking to the curriculum design of an emerging transdisciplinary engineering program for digital manufacturing.

\section{HOW: Design methodology within the current engineering curricula}

Design methodology is defined as a set of design methods/tools that augment design thinking. The engineering schools and design schools both claim to utilize design methodology in their practices. The roots are slightly different. In a full picture of the product development lifecycle that covers from the demand side to the supply side, stakeholders get involved in various stages about design, development, part production, assembly, and maintenance support. There has been widely recognized design methodology such as function analysis, quality function deployment and BoothroydDewhurst's design for manufacturing and assembly. They consider single or multiple stages of the product development lifecycle. Some focus on reducing part counts and assembly operations to minimize the production cost, and others attempt to identify implicit requirements and needs for greater customer satisfaction. Regardless of qualitative or quantitative approaches, design methodology facilitates the desirable design outcome for efficiency, sustainability, creativity etc. Next, we would like to highlight a few examples of how design thinking (as a method) has benefited engineering innovations within the current engineering curriculum.

\subsection{Sketches, drawings, and design thinking workshop}

Sketching is one of the most intuitive design methodologies. It can convey conceptual ideas, visualize the product functionality and human interaction. Unlike engineering drawings, which is more detailed on actual geometry and proportional dimension, sketching is handy for exploring the form. Therefore, this method is still relevant and crucial in today's practice of individual designers. On the other hand, design thinking workshops built upon a collaborative mindset, similar to the concept of co-design and participatory design. A design thinking workshop is held to gather insights by putting a diverse variety of people together to address the given topic. Everyone will brainstorm to reframe the problem statement collectively and broaden the innovative ideas. The 
classical design thinking exercises sometimes are criticized for ending with just a room full of Post-Its. It is important to note that the inquiry nature of design thinking brings in the creation of systems.

\subsection{Knowledge-based design systems}

When scientists begin to discuss the possibility of AI for design, a research stream on knowledge-based design systems rises. It is a computer-aided program that supports decision-making for complex problems. Specifically, the design procedures are captured as a set of algorithms and design rules. Take a golf club design for example. Once we computerize the correlations between design performance requirement (wind resistance) and product properties (curve), numerous 3D model variations can be generated automatically [11]. Such systems realize a seamless integrated approach to reduce timeto-market from digital design to manufacturing.

In addition to the improvement in engineering performance (e.g., weight, strength), we can leverage generative design algorithms to explore complex geometric forms. For instance, it brings the potential of biomimicry to produce organic shapes inspired by nature. The fabrication of these cellular and lattice structures becomes possible due to the additive manufacturing (AM) process. After hundreds of thousands of conceptual designs are generated, some of them may have comparable functional performance. In this case, what are the final selection criteria to go into the production phase? Can AI make the products appeal more to the customers?

Considering that emotional design add value to products and services, we can build a knowledge-based design system for users' psychological needs. Kansei engineering is a philosophy that aims at translating subjective feelings into product specifications [12]. Kansei is a Japanese term meaning human emotions, feelings and impressions towards a product or service. Kansei can be quantified in either the physiological level (eye movement, facial expression) or the semantic level (semantic differential scale). In this regard, researchers have developed a Kansei engineering system (KES) from different perspectives.

$\mathrm{Li}$, et al. [13] applied multi-objective optimization to satisfy user's affective responses for vase forms. Although beauty is in the eyes of the beholder, there are some ground rules for visual perceptions such as most people prefer symmetry. In light of this, Lugo, et al. [14] exploited the gestalt principles to generate the design of wheel rims. Lastly, it worth noting that the visually appealing style is just one application of users' feelings. Knowledge-based KES can push further to enable user experience study such as building trust in autonomous vehicle safety [15].

\section{Design for transdisciplinary engineering education}

With the advancement in information technology, the impact of design thinking is visible in many digital engineering tools. Modern devices connect to the Internet, generate, and collect usage activity data continuously. Engineers can now employ AI to understand users, machines, systems, and organizations in a bid to assist real-time decision-making. On the whole, the trend of design informatics is an embodiment of design thinking and data science. Relevant machine learning techniques have been proposed to manage such heterogeneous data, make them informative, then acquire contextual knowledge. 


\subsection{Trend in the cyberphysical age}

Design thinking has driven the evolution of design methodology research. The early mobile phones have various forms, colorful buttons, and even flip-style designs [16]. Today, those mechanical features have been replaced by the touch screen [17]. The increasing screen size indicates that users spend a great amount of time on the digital interface. The digitalization of design methodology, therefore, thrives under the umbrella term of user experience (UX). Moreover, the focus of design development shifts from product to service. While everything is going virtual, it is of importance to consider cyberphysical interactions in the real world. One seminal example of human-machine interaction is the hand gestures that adjust the size of the digital photo on the touch screen. Think about how we used to interact with the TV. At first, you must press the button on a physical TV box before the remote controls were invented. Now, one device can communicate to all home appliances at once and is even accessible using voice control. In short, disruptive technologies are clearly changing our everyday experience.

As AI continues to advance, machines are getting smarter. They can make recommendations after learning your behavior patterns such as your most frequent routes when travelling via taxi and the items you are likely to purchase based on your activities. These models are trained and perform best to predict events based on the data you feed in. However, we argue that AI for design may not be able to think out of the 'cyber box' nor stress enough the importance of physical domains to create an impactful user experience. In the cyberphysical age, design thinking does not necessarily demand sophisticated technology. For instance, the Wii was popular at a time because the designers relooked into simple motion sensors then proposed an intuitive way to interact with digital games. It is evident that an emphasis on UX is warranted in this era of cyberphysical interactions.

\subsection{Holistic curriculum design for digital manufacturing}

Digital manufacturing is an adequate example of this cyberphysical integration [18]. However, the current educational coursework materials in the mainstream engineering curricula mainly focus on scientific principles and rules in one domain. They are not ready for providing a holistic perspective to the students via a system-of-system approach for an interdisciplinary engineering concept. To the best of our knowledge, this paper is the first to explore the application of design thinking in an engineering curriculum for digital manufacturing. This curriculum should prepare the future workforce for the latest challenges of the manufacturing landscape, and train them to innovatively devise better UX in this cyberphysical age. With that goal, we propose a comprehensive book series that breakdown digital manufacturing into three aspects: design, device, and digital factory.

The first volume will look at the design aspect from digital design to part manufacturing in the context of 3D printing. The design methodologies will be adopted to exploit the full potentials of additive manufacturing. The second volume focuses on the devices and agents at the factory level such as industrial Internet of things, digital twins, and cyberphysical security. These applications inform a complex multi-input multi-output system for predictive modeling. The second book also presents new business models working towards a sustainable net zero operations and economy. The digital architecture enables greater resilience for supply chain management such as the unexpected demand shifts during the COVID-19 pandemic. 
Indeed, there are already many existing textbooks on the market for the abovementioned individual topics. The main contribution of our curriculum is to put leading experts from various fields together to tell one cohesive story. It will incorporate the major aspects of 3D printing with immense depth in science and engineering fundamentals, and breadth in a range of technologies. This enables the readers to have a bigger picture knowing how different disciplines work together to fulfill the vision of advancing towards digital manufacturing. In this sense, design thinking is an overall method to describe our approach in designing an engineering curriculum for digital manufacturing. We believe a holistic curriculum design is essential for the future of transdisciplinary engineering education.

\section{Conclusion}

This paper aims to discuss the application of design thinking in transdisciplinary engineering education. Rather than debating on its vague definition, we used explicit examples to depict the role of design thinking in current engineering schools and design schools. Evidence from knowledge-based design systems has demonstrated the advantages and limitations of AI design methodology in engineering innovations. Therefore, we expect that design thinking skills will remain pivotal when it comes to creativity and curriculum development. Moving towards the cyberphysical age, the workforce of the future must obtain a broad base of general skills and knowledge that enhance collaborations across the disciplines. Consequently, a creative curriculum that lay the ground for digital manufacturing in the engineering school is warranted.

\section{Acknowledgement}

This paper is supported by HP-NTU Digital Manufacturing Corporate Lab (School of Mechanical \& Aerospace Engineering, Nanyang Technological University), National Research Foundation (NRF) Singapore and the Singapore Government through the Industry Alignment Fund - Industry Collaboration Projects Grant (I1801E0028).

\section{References}

[1] Y. Li, A. H. Schoenfeld, A. C. Graesser, L. C. Benson, L. D. English, and R. A. Duschl, Design and Design Thinking in STEM Education, Journal for STEMEducation Research, 2019, Vol. 2, pp. 93-104,

[2] N. Wognum, J. Mo and J. Stjepandić, Transdisciplinary Engineering Systems, R. S. Kenett, R. S. Swarz, and A. Zonnenshain (Eds.) Systems engineering in the fourth industrial revolution: Big data, novel technologies, and modern systems engineering, John Wiley \& Sons, 2019, pp 483-510.

[3] C. M. Gray, C. M. Seifert, S. Yilmaz, S. R. Daly and R. Gonzalez, What is the content of "design thinking"? Design heuristics as conceptual repertoire, International Journal of Engineering Education, 2016, Vol. 32, no. 3B, pp. 1349-1355.

[4] W. Brenner, F. Uebernickel and T. Abrell, Design thinking as mindset, process, and toolbox, in W. Brenner, F. Uebernickel, Design thinking for innovation: Research and Practice, Springer Nature Switzerland, 2016, pp. 3-21.

[5] C. Dai, C. C. Wang, C. Wu, S. Lefebvre, G. Fang, and Y.-J. Liu, Support-free volume printing by multi-axis motion, ACM Transactions on Graphics (TOG), 2018, Vol. 37, No. 4, pp. 1-14.

[6] C. L. Dym, A. M. Agogino, O. Eris, D. D. Frey and L. J. Leifer, Engineering design thinking, teaching, and learning, Journal of engineering education, 2005, Vol. 94, No. 1, pp. 103-120. 
[7] E. Coleman, T. Shealy, J. Grohs, and A. Godwin, Design thinking among first-year and senior engineering students: A cross-sectional, national study measuring perceived ability, Journal of Engineering Education, 2020, Vol. 109, No. 1, pp. 72-87.

[8] R. M. Clark, L. M. Stabryla, and L. M. Gilbertson, Sustainability coursework: student perspectives and reflections on design thinking, International Journal of Sustainability in Higher Education, 2020, Vol. 21, No. 3, pp. 593-611.

[9] M. Palacin-Silva, J. Khakurel, A. Happonen, T. Hynninen, and J. Porras, Infusing design thinking into a software engineering capstone course, 2017 IEEE 30th Conference on Software Engineering Education and Training (CSEE\&T), 2017: IEEE, pp. 212-221.

[10] Y. Tomita, K. Watanabe, S. Shirasaka, and T. Maeno, Applying design thinking in systems engineering process as an extended version of DIKW model, INCOSE International Symposium, 2017, Vol. 27, No. 1, pp. 858-870.

[11] D. Chang and C.-H. Chen, Digital design and manufacturing of wood head golf club in a cyber physical environment, Industrial Management \& Data Systems, 2017, Vol. 117, No. 4, pp. 648-671.

[12] M. Nagamachi and A. M. Lokman, Innovations of Kansei engineering. CRC Press, Boca Raton, 2016.

[13] Y. Li, M.-D. Shieh, and C.-C. Yang, A posterior preference articulation approach to Kansei engineering system for product form design, Research in Engineering Design, 2019, Vol. 30, No. 1, pp. 3-19.

[14] J. E. Lugo, J. P. Schmiedeler, S. M. Batill, and L. Carlson, Relationship between product aesthetic subject preference and quantified Gestalt principles in automobile wheel rims, Journal of Mechanical Design, 2016, Vol. 138, No. 5, 051101.

[15] K. Kaur and G. Rampersad, Trust in driverless cars: Investigating key factors influencing the adoption of driverless cars, Journal of Engineering and Technology Management, 2018, Vol. 48, pp. 87-96.

[16] J. R. Jiao, Y. Zhang, and M. Helander, A Kansei mining system for affective design, Expert Systems with Applications, 2006, Vol. 30, No. 4, pp. 658-673.

[17] K. Y. Chan, C. Kwong, T. Dillon, and K. Fung, An intelligent fuzzy regression approach for affective product design that captures nonlinearity and fuzziness, Journal of Engineering Design, 2011, Vol. 22, No. 8, pp. 523-542.

[18] C. D. Patel, Cyberphysical Integration, The Bridge, 2020, Vol. 50, No. 4, pp. 21-23. 